\title{
Propriedades térmicas de compósitos de acrilonitrila-butadieno-estireno (ABS) e fibras de celulose modificadas com nanopartículas de sílica $\left(\mathrm{SiO}_{2}\right)$
}

\section{Thermal properties of acrylonitrile-butadiene- styrene (ABS) composites reinforced with silica nanoparticles $\left(\mathrm{SiO}_{2}\right)$ modified cellulose}

\author{
Emanuela Camargo de Barros Lustosa ${ }^{1}$, Cláudio Henrique Soares Del Menezzi ${ }^{1}$, \\ Sandra Maria da Luz ${ }^{2}$, Joabel Raabe ${ }^{1}$, Luiz Carlos Correia de Jesus ${ }^{3}$, \\ Rosineide Miranda Leão ${ }^{2,4}$, Ademir José Zattera ${ }^{5}$
}

\footnotetext{
${ }^{1}$ Departamento de Engenharia Florestal, Faculdade de Tecnologia - Universidade de Brasília, CEP: 70910-900, Brasília, DF, Brasil.

${ }^{2}$ Faculdade do Gama - Universidade de Brasília, CEP: 72444-240, Brasília, DF, Brasil.

${ }^{3}$ Departamento de Eng. Mecânica, Faculdade de Tecnologia - Universidade de Brasília, CEP: 70910-900, Brasília, DF, Brasil.

${ }^{4}$ Universidade Paulista de Brasília, Instituto de Ciências Exatas e Tecnologia, CEP: 70390-130, Brasília, DF, Brasil.

${ }^{5}$ Centro de Ciências Exatas e Tecnologia, Universidade de Caxias do Sul, CEP: 95070-560, Caxias do Sul, RS, Brasil.

e-mail: emanuelacblustosa@gmail.com,cmenezzi@unb.br
}

\section{RESUMO}

O objetivo deste trabalho foi caracterizar os compósitos produzidos com o terpolímero acrilonitrila-butadieno-estireno (ABS) reforçados com fibras de polpa celulose modificadas com nanopartículas de sílica $\left(\mathrm{SiO}_{2}\right)$. Primeiramente, as fibras foram modificadas utilizando o processo sol-gel, tendo como precursor da sílica o ortosilicato de tetraetila (TEOS). Adicionalmente, duas concentrações de TEOS e dois tempos de reação foram utilizados para produzir dois tipos de fibras modificadas, $\mathrm{F}_{\mathrm{T} 1}$ e $\mathrm{F}_{\mathrm{T} 2}$, logo depois, foi determinado o rendimento de cada processo de modificação. As fibras foram caracterizadas por meio da microscopia eletrônica de varredura (MEV), análise termogravimetria (TGA) e sua derivada (DTG). Depois da obtenção das fibras os compósitos de acrilonitrila-butadieno-estireno reforçados com 10 e $20 \%(\mathrm{~m} / \mathrm{m})$ de fibras de polpa celulose branqueada não modificadas $\left(\mathrm{F}_{\mathrm{NT}}\right)$ e modificadas com nanopartículas de sílica $\left(\mathrm{F}_{\mathrm{T} 1}\right.$ e $\left.\mathrm{F}_{\mathrm{T} 2}\right)$ foram obtidos por extrusão e posteriormente por injeção, depois os compósitos foram caracterizados quanto à densidade, análise térmica (TGA/DTG), temperatura de deflexão térmica (HDT) e a temperatura de amolecimento VICAT. A adição de fibras, de modo geral, proporcionou incremento para a temperatura de deflexão térmica HDT e temperatura de amolecimento VICAT, sendo mais acentuado quando o teor de fibras passou de $10 \%$ para $20 \%$. No entanto, o aumento do teor de fibras de $10 \%$ para $20 \%$, em comparação ao ABS puro provocou diminuição da temperatura de início de degradação e provocou aumento da densidade.

Palavras-chave: compósitos, celulose, nanopartículas de sílica, sol-gel, acrilonitrila-butadieno-estireno.

\section{ABSTRACT}

The aim of this study was to evaluate thermal properties of composites made with terpolymer acrylonitrile-butadienestyrene (ABS) reinforced with silica nanoparticles $\left(\mathrm{SiO}_{2}\right)$ modified cellulose. Primarily, the fibers were modified using the sol-gel process and the tetraethyl ortosilicate (TEOS) as precursor. In addition, two concentrations of TEOS and two reaction times were used to produce two types of modified fibers, $\mathrm{F}_{\mathrm{T} 1}$ and $\mathrm{F}_{\mathrm{T} 2}$ fibers, then was determined the yield of modification process. The fibers were characterized by scanning electron microscopy (SEM), thermogravimetric analysis (TGA) and its derivative (DTG). The acrylonitrile-butadiene-styrene composites reinforced with 10 and $20 \%$ (w/w) unmodified cellulose pulp fibers $\left(\mathrm{F}_{\mathrm{NT}}\right)$ and silica nanoparticles modified $\left(\mathrm{F}_{\mathrm{T} 1}\right.$ and $\mathrm{F}_{\mathrm{T} 2}$ ) were obtained by extrusion process 
and subsequent injection. The composites were characterized in terms of density, thermogravimetric (TGA / DTG), thermal deflection temperature (HDT) and VICAT softening temperature. In general, the addition of fibers provided an increase for HDT and VICAT, being more pronounced when the fiber content increased from $10 \%$ to $20 \%$. However, in comparison with ABS the increasing of fiber content from $10 \%$ to $20 \%$, caused a decrease in $\mathrm{T}_{\text {onset }}$ and caused an increase in density.

Keywords: composites, cellulose, silica nanoparticles, sol-gel, acrylonitrile-butadiene-styrene.

\section{INTRODUÇÃO}

A atual situação ambiental estimula o aumento da demanda pelo desenvolvimento de materiais que sejam mais sustentáveis e que satisfaçam as propriedades requeridas. A produção de compósitos poliméricos utilizando fibras naturais como material de reforço é uma boa alternativa aos materiais convencionais.

A matriz é responsável por transferir a tensão entre as fibras. É através da região interfacial que os esforços atuantes na matriz são transferidos ao material de reforço, e a eficiência dessa transferência de tensão está diretamente relacionada com a qualidade da ligação fibra-matriz. Além disso, a matriz providencia uma barreira de proteção contra as adversidades do meio e protege a superfície das fibras contra a abrasão mecânica [1-3]. O reforço/fase dispersa contribui para o aprimoramento adicional das propriedades do material [4].

Os compósitos tradicionalmente são confeccionados com fibras de alta resistência como carbono, vidro e aramida e matrizes poliméricas. No entanto, essas fibras apresentam sérias desvantagens, como: não são renováveis, não recicláveis, alto consumo de energia para sua obtenção, risco para a saúde quando inaladas e não são biodegradáveis [5]. Os compósitos em que o material de reforço é constituído por fibras naturais são ambientalmente mais interessantes e podem ser utilizados em inúmeras aplicações, como no setor automotivo, aeroespacial, construção civil, embalagens, entre outros [6].

O interesse por compósitos poliméricos reforçados com fibras naturais em termos de indústria e de pesquisa tem crescido muito nos últimos anos. Diversas fontes naturais de fibras têm sido estudadas e aplicadas como material de reforço em compósitos de matrizes poliméricas, como por exemplo o algodão, kenaf, cânhamo, juta, sisal, bambu, banana, abacaxi, madeira, entre outros [6]. Adicionalmente, o sucesso do emprego destes tipos de compósitos depende das propriedades inerentes as fibras e a matriz, bem como da compatibilidade entre estes componentes [7].

Nesse sentido, uma das limitações do uso de fibras naturais é a natureza polar e hidrofílica das fibras lignocelulósicas e as características apolares da maioria dos polímeros convencionais, o que causa incompatibilidade entre a fibra e a matriz, e resulta em dificuldade para atingir um elevado nível de dispersão do material de reforço dentro da matriz polimérica, proporcionando uma fraca adesão interfacial [8]. Concomitantemente, uma das alternativas para melhorar a compatibilidade das fibras com a matriz polimérica é a incorporação química de agentes de acoplamento às fibras vegetais. Além disso, há também a possibilidade de realizar modificações físico-químicas na superfície das fibras [9].

Uma das alternativas para contornar essa limitação consiste na modificação da superfície das fibras, como por exemplo a deposição de nanopartículas de sílica $\left(\mathrm{SiO}_{2}\right)$ na superfície das fibras de polpa celulose através do processo solgel gerando um material híbrido orgânico-inorgânico com distintas propriedades e aplicações. Raabe et al. [10] observaram que a modificação das fibras de polpa celulose pela deposição de nanopartículas de sílica favoreceu a estabilidade térmica das fibras e a resistência a umidade. Com isso, surge a oportunidade de testar diferentes aplicações para essas fibras modificadas, sendo interessante avaliar sua aplicação em compósitos poliméricos. Na atualidade, grande parte da celulose é destinada para a confecção de pasta para papel, já os materiais híbridos de celulose-sílica, devido às suas propriedades, se mostram promissores para diferentes aplicações [11].

Os polímeros mais comumente utilizados como matriz para a fabricação de compósitos são os termoplásticos. Entre os termoplásticos tem-se o terpolímero acrilonitrila-butadieno-estireno (ABS), que é um dos principais polímeros de engenharia e apresenta diversas aplicações, como no setor automobilístico, eletrônicos e eletrodomésticos [12]. Neste contexto, o objetivo deste trabalho foi caracterizar compósitos confeccionados com o acrilonitrila-butadieno-estireno (ABS) reforçados com fibras de polpa celulose modificadas com nanopartículas de sílica $\left(\mathrm{SiO}_{2}\right)$.

\section{MATERIAIS E MÉTODOS}

\subsection{Modificação das Fibras}

Foram utilizadas fibras de polpa celulose de Eucalyptus sp. oriundas da indústria com teor de $\alpha$-celulose de 91,08\%, fornecidas pela Eldorado Brasil Celulose. As fibras passaram pelo processo de modificação utilizando a metodologia aplicada por Raabe et al. [10], onde aplicou-se dois tratamentos: o primeiro tratamento $\left(\mathrm{F}_{\mathrm{T} 1}\right)$ consistiu em tempo de 2 horas e concentração do precursor TEOS de 1,9 g. $\mathrm{g}^{-1}$, o segundo tratamento $\left(\mathrm{F}_{\mathrm{T} 2}\right)$ aplicou-se tempo de 18 horas e a concentração de precursor TEOS de 4,2 g. $\mathrm{g}^{-1}$. O processo consistiu na imersão de fibras de polpa celulose em uma solução com catalisador 
de síntese o hidróxido de amônia $\left(\mathrm{NH}_{4} \mathrm{OH}-30 \%\right.$ v. $\left.\mathrm{v}^{-1}\right)$, solvente etanol $\left(\mathrm{CH}_{3} \mathrm{CH}_{2} \mathrm{OH}-95 \%\right.$ P.A.) e água destilada. Essa solução permaneceu em agitação (300 rpm) por 2 horas, após esse tempo, o precursor ortosilicato de tetraetila $\left(\mathrm{C}_{8} \mathrm{H}_{20} \mathrm{O}_{4} \mathrm{Si}\right.$ - TEOS, 98\%), o TEOS foi adicionado lentamente na solução. Após transcorrido o tempo estipulado, a solução foi então filtrada e as fibras lavadas com água destilada, e depois as fibras foram encaminhadas para a secagem em estufa a $70 \pm 5$

${ }^{\circ} \mathrm{C}$, o processo de modificação foi realizado à temperatura ambiente.

Para a modificação da fibra de polpa celulose $\mathrm{F}_{\mathrm{T} 1}$ foram utilizadas as seguintes proporções: etanol = 87,5\%; hidróxido de amônia $=1,5 \%$; água destilada $=9 \%$ e TEOS $=2 \%$. E para a fibra de polpa celulose $\mathrm{F}_{\mathrm{T} 2}$ foram aplicadas as seguintes proporções: etanol $=85 \%$; hidróxido de amônia $=1,5 \%$; água destilada $=9 \%$ e TEOS $=4,5 \%$. As fibras de polpa celulose foram adicionadas à solução respeitando a proporção de $1: 100 \mathrm{~g} \cdot \mathrm{mL}^{-1}$.

\subsection{Determinação do rendimento da modificação das fibras}

Foram avaliados os rendimentos dos processos de modificação das fibras, utilizando balança de precisão $(0,0001 \mathrm{~g})$ para realizar a pesagem da quantidade de fibras que foi inserida na mistura e posteriormente para pesar as fibras já modificadas e secas em estufa. $\mathrm{O}$ rendimento foi obtido por diferença de massa conforme Equação 1.

$$
R=\frac{(M f-M i)}{M i} \cdot 100
$$

Onde:

$\mathrm{R}=$ rendimento $(\%)$

Mf = Massa final $(\mathrm{g})$

$\mathrm{Mi}=$ Massa inicial $(\mathrm{g})$

\subsection{Microscopia eletrônica de varredura (MEV) das fibras}

A análise de microscopia eletrônica de varredura foi realizada utilizando o MEV modelo JSM - 7001F da marca JEOL. Primeiramente, ocorreu o recobrimento duplo com ouro do material. Foi utilizado um detector de elétrons secundários e o equipamento operou com uma aceleração de $5 \mathrm{kv}$.

\subsection{Produção dos compósitos}

O polímero utilizado como matriz para a produção dos compósitos foi o acrilonitrila-butadieno-estireno (ABS) AF3500 Tairilac ABS de alta fluidez da empresa Flamel Polímeros, indicado para aplicação em eletrônicos, brinquedos, eletrodomésticos e puxadores de portas de geladeira.

Foram confeccionados compósitos utilizando como matriz o polímero ABS e como reforço as fibras de polpa celulose não tratadas $\left(\mathrm{F}_{\mathrm{NT}}\right)$, fibras submetidas ao tratamento $1\left(\mathrm{~F}_{\mathrm{T} 1}\right)$ e as fibras submetidas ao tratamento $2\left(\mathrm{~F}_{\mathrm{T} 2}\right)$ para cada tipo de fibra foram aplicadas duas proporções de fibra na matriz sendo $10 \%$ e $20 \%(\mathrm{~m} / \mathrm{m})$, o ABS foi considerado a testemunha, com a qual as propriedades obtidas foram comparadas: $\mathrm{ABS}, \mathrm{ABS} / \mathrm{F}_{\mathrm{NT} .10 \%}, \mathrm{ABS} / \mathrm{F}_{\mathrm{NT} .20 \%}, \mathrm{ABS} / \mathrm{F}_{\mathrm{T} 1.10 \%}, \mathrm{ABS} /$ $\mathrm{F}_{\mathrm{T} 1.20 \%}, \mathrm{ABS} / \mathrm{F}_{\mathrm{T} 2.10 \%}, \mathrm{ABS} / \mathrm{F}_{\mathrm{T} 2.20 \%}$.

Antes da confecção dos compósitos os materiais (fibras e ABS) foram previamente secos em estufa por $24 \mathrm{~h}$ a temperatura de $70 \pm 5^{\circ} \mathrm{C}$. Após a secagem, o polímero ABS e as fibras, nas suas devidas proporções foram levados ao processamento em extrusora dupla-rosca de alimentação dupla e com oito zonas de aquecimento $\left(179{ }^{\circ} \mathrm{C}, 177^{\circ} \mathrm{C}, 200\right.$ ${ }^{\circ} \mathrm{C}, 200{ }^{\circ} \mathrm{C}, 200{ }^{\circ} \mathrm{C}, 200{ }^{\circ} \mathrm{C}, 200{ }^{\circ} \mathrm{C}, 160{ }^{\circ} \mathrm{C}$ ) onde ocorreu a mistura do material (ABS/Fibra). O material misturado foi extrudado na forma de "espaguete" e, posteriormente, transformado em "pellets", em seguida, o material seguiu para a máquina injetora utilizando três zonas de aquecimento $\left(200^{\circ} \mathrm{C}, 180^{\circ} \mathrm{C}\right.$ e $\left.170{ }^{\circ} \mathrm{C}\right)$, com velocidade de rotação da rosca de $70 \mathrm{rpm}$.

\subsection{Termogravimetria (TGA/DTG) das fibras, ABS e compósitos}

Para a realização da análise foi utilizado um analisador simultâneo TGA-DSC da TAInstruments, modelo Q600, com uma massa aproximada de $10 \mathrm{mg}$ de amostra em um cadinho de alumina, submetidas numa faixa temperatura de 30 a $600{ }^{\circ} \mathrm{C}$, com rampa de aquecimento de $10^{\circ} \mathrm{C} \cdot \mathrm{min}^{-1}$ sob atmosfera de $\mathrm{N}_{2}$ (fluxo de $100 \mathrm{~mL} \cdot \mathrm{min}^{-1}$ ). 


\subsection{Determinação da densidade dos compósitos}

A densidade foi determinada conforme norma ASTM D792 - 13 (ASTM, 2013). Foram utilizados quatro corpos de prova para cada tratamento. As amostras foram pesadas em balança analítica $(0,0001 \mathrm{~g})$ e as amostras foram imersas em etanol.

\subsection{Temperatura de deflexão térmica (HDT) e temperatura de amolecimento VICAT do ABS e compósitos}

Os ensaios de temperatura de deflexão térmica (HDT) e temperatura de amolecimento VICAT foram realizados em equipamento CEAST, modelo HDT/6VICAT (Figura 1). Foram utilizados três corpos de prova de cada tratamento para HDT e três corpos de prova de cada tratamento para VICAT.

A temperatura de deflexão térmica (HDT) foi determinada conforme a norma ASTM D648-07 (ASTM, 2004), com taxa de aquecimento de $120^{\circ} \mathrm{C} / \mathrm{h}$ e carga aplicada no material de $1,82 \mathrm{MPa}$. No ensaio os corpos de prova foram submersos em banho de óleo de silicone e permaneceram sob efeito da carga até sofrerem um deslocamento vertical igual a $0,25 \mathrm{~mm}$. O ponto de amolecimento VICAT foi determinado pela norma ASTM D1525 - 09 (ASTM, 2009) com taxa de aquecimento de $120^{\circ} \mathrm{C} / \mathrm{h}$ e carga aplicada de $10 \mathrm{~N}$. No ensaio os corpos de prova foram submersos em banho de óleo de silicone e a agulha de ponta plana deve penetrar $1 \mathrm{~mm}$ no corpo de prova.

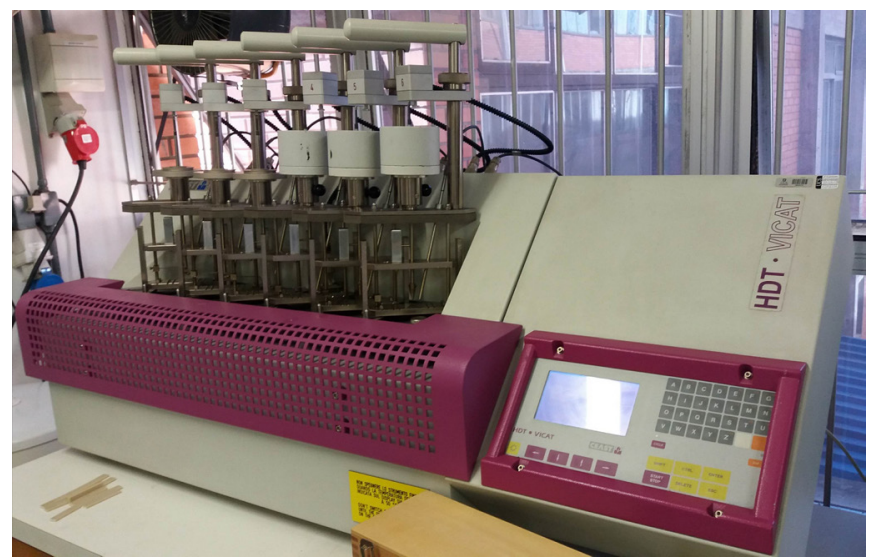

Figura 1: Equipamento para realização do ensaio HDT/VICAT.

\section{RESULTADOS E DISCUSSÃO}

\subsection{Determinação do rendimento da modificação das fibras}

As fibras de polpa celulose utilizadas, por serem oriundas da indústria, já são resultado do processo de polpação e branqueamento, então o rendimento apresentado corresponde apenas ao processo de modificação das fibras com a adição de nanopartículas de sílica.

Durante o processo de modificação da superfície das fibras houve ganho de massa referente à adição das nanopartículas de sílica à superfície das fibras. A fibra submetida ao tratamento $2\left(\mathrm{~F}_{\mathrm{T} 2}\right)$, apresentou um incremento em massa de 40,09 \%, sendo maior do que o incremento da fibra submetida ao tratamento $1\left(\mathrm{~F}_{\mathrm{T} 1}\right)$, que apresentou incremento de $20,55 \%$. O maior ganho de massa apresentado pela $\mathrm{F}_{\mathrm{T} 2}$ está diretamente relacionado com a concentração do precursor TEOS e o tempo de reação, visto que no tratamento 2 a concentração do precursor TEOS era mais alta $\left(4,2 \mathrm{~g} \cdot \mathrm{g}^{-1}\right)$ e o tempo de reação mais longo (18 h) o que proporcionou uma maior agregação das nanopartículas de sílica na superfície das fibras de polpa celulose, resultando no ganho de massa do material.

\subsection{Microscopia eletrônica de varredura (MEV) das fibras}

A Figura 2 apresenta as micrografias eletrônicas de varredura das fibras de polpa celulose $\mathrm{F}_{\mathrm{NT}}, \mathrm{F}_{\mathrm{T} 1}$ e $\mathrm{F}_{\mathrm{T} 2}$. Foi observado na Figura 2a uma superfície rugosa típica de celulose, e as Figuras $2 \mathrm{~b}$ e c indicam as fibras modificadas $\left(\mathrm{F}_{\mathrm{T} 1}\right.$ e $\left.\mathrm{F}_{\mathrm{T} 2}\right)$, mostrando uma boa distribuição das nanopartículas de sílica na superfície da fibra de celulose de forma homogênea, assim, apresentando a eficiência da modificação da superfície da fibra de celulose. Na superfície da fibra $\mathrm{F}_{\mathrm{T} 1}$ observa-se partículas de sílica individualizadas, conforme mostra as setas. Já na superfície da fibra $\mathrm{F}_{\mathrm{T} 2}$ verifica-se um recobrimento mais espesso sendo difícil a visualização de partículas isoladas.

O comportamento apresentado foi similar ao observado por Raabe et al.[10], em que as amostras contendo menor concentração de TEOS $\left(1,9\right.$ g. $\left.\mathrm{g}^{-1}\right)$ com tempo de reação de 2 e 12 horas apresentaram boa dispersão e partículas 
individualizadas na superfície das fibras e para a amostra com maior concentração de TEOS $\left(8,4\right.$ g.g $\left.\mathrm{g}^{-1}\right)$ e tempo de reação de 18 horas verificaram que as fibras apresentaram uma camada de revestimento mais uniforme, com quase nenhum espaço vazio entre as nanopartículas de sílica, indicando que concentração do precursor TEOS e o tempo de reação influenciaram a distribuição e o tamanho das partículas de sílicas depositadas sobre a superfície das fibras.

Pinto et al. [13] ao realizarem a modificação de fibras de celulose a partir do método sol-gel utilizando o TEOS como precursor também observaram a formação de uma película densa de sílica sobre as fibras de celulose.
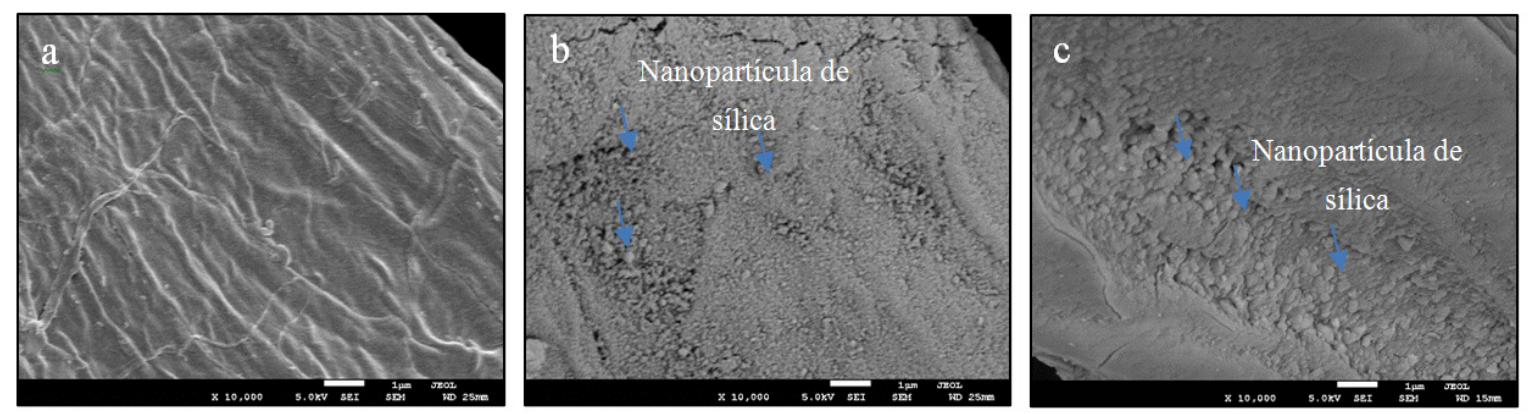

Figura 2: Micrografias das fibras de polpa celulose: a) fibra não tratada $\left(\mathrm{F}_{\mathrm{NT}}\right)$; b) fibra com nanopartículas de sílica $\left(\mathrm{F}_{\mathrm{T} 1}\right)$; c) fibra com nanopartículas de sílica $\left(\mathrm{F}_{\mathrm{T} 2}\right)$.

\subsection{Termogravimetria (TGA/DTG) das fibras e dos compósitos}

As fibras foram analisadas por análise termogravimétrica. Esta análise é utilizada para avaliar o nível de estabilidade térmica da amostra. A estabilidade térmica ficou na faixa de temperatura entre $305^{\circ} \mathrm{C}$ para a $\mathrm{F}_{\mathrm{NT},} 318{ }^{\circ} \mathrm{C}$ para a $\mathrm{F}_{\mathrm{T} 1}$ e $320{ }^{\circ} \mathrm{C}$ para a $\mathrm{F}_{\mathrm{T} 2}$. Logo, a modificação da superfície das fibras com nanopartículas de sílica contribuiu para a melhoria estabilidade térmica das fibras, visto que aumentou a temperatura de início de degradação $\left(\mathrm{T}_{\text {onsee }}\right)$ das fibras tratadas com relação a $\mathrm{F}_{\mathrm{NT}}$, com maior destaque para a $\mathrm{F}_{\mathrm{T} 2}$, visto que sua $\mathrm{T}_{\text {onset }}$ foi $15^{\circ} \mathrm{C}$ superior à $\mathrm{T}_{\text {onset }}$ da $\mathrm{F}_{\mathrm{NT}}$, resultado similar ao encontrado por Raabe et al.[10].

Com base na curva TGA (Figura 3a) com relação à quantidade de resíduo a $600{ }^{\circ} \mathrm{C}$ observa-se que a $\mathrm{F}_{\mathrm{T} 2}$ apresentou o maior resíduo ( 40,9\%) quando comparada com as outras fibras, $\mathrm{F}_{\mathrm{NT}}(\sim 13,1 \%)$ e $\mathrm{F}_{\mathrm{T} 1}(\sim 27,7 \%)$. Segundo Yang et al. [14], quando a temperatura ultrapassa os $400{ }^{\circ} \mathrm{C}$ praticamente toda a celulose é degradada, apresentando pouca massa residual. Observou-se que a modificação das fibras de polpa celulose acarretou em aumento da massa residual, o que está diretamente relacionado com a quantidade de partículas inorgânicas de sílica presentes nas fibras tratadas [10], estando relacionado com o rendimento do processo de modificação das fibras de polpa celulose, visto que, a $\mathrm{F}_{\mathrm{T} 1}$ apresentou um incremento em massa de $\sim 20 \%$ e a $\mathrm{F}_{\mathrm{T} 2}$ incremento de $\sim 40 \%$, indicando que a massa residual é basicamente composta pelas nanopartículas de sílica e que a fibra $\mathrm{F}_{\mathrm{T} 2}$ teve maior agregação de nanopartículas de sílica à sua superfície.
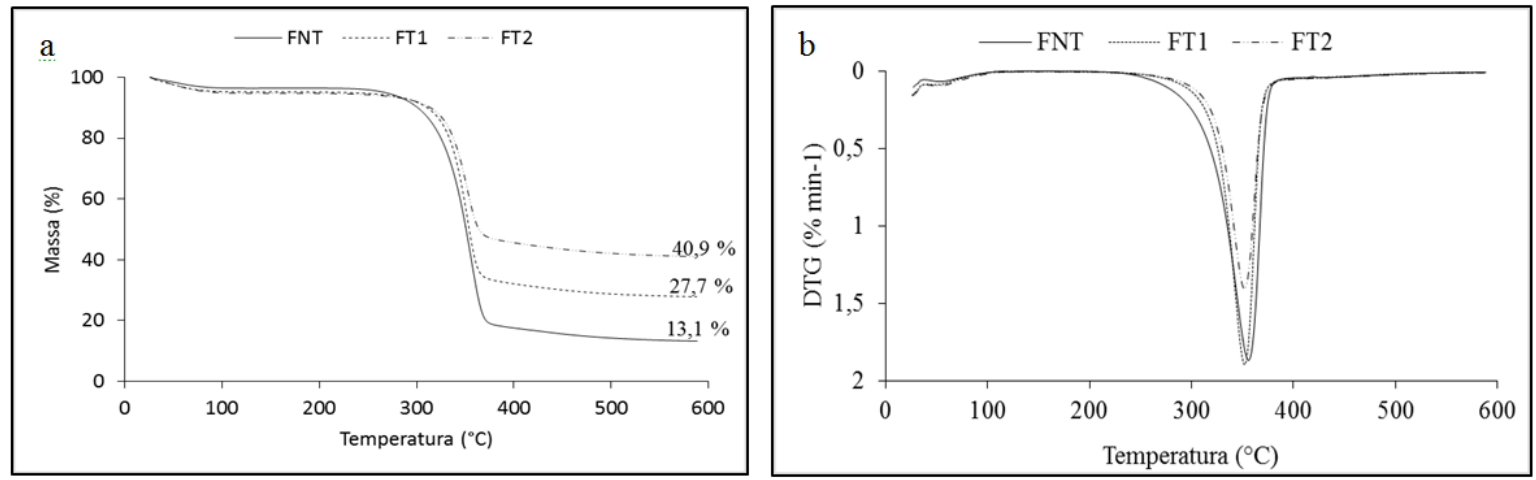

Figura 3: a) Curvas TGA; b) curvas DTG para as fibras não tratadas $\left(\mathrm{F}_{\mathrm{NT}}\right)$, tratamento $1\left(\mathrm{~F}_{\mathrm{T} 1}\right)$ e tratamento $2\left(\mathrm{~F}_{\mathrm{T} 2}\right)$.

Na Tabela 1 são apresentados os valores percentuais de perda de massa para as temperaturas de 100, 200, 300, 400 e $500{ }^{\circ} \mathrm{C}$ e a temperatura na qual a taxa de velocidade de variação é máxima $\left(\mathrm{T}_{\text {pico }}\right)$. Observa-se que as fibras apresentaram valores similares com $\mathrm{T}_{\text {pico }}$ variando de $352^{\circ} \mathrm{C}$ a $355^{\circ} \mathrm{C}$. De acordo com Yang et al. [14], entende-se que a perda de massa na faixa até $\sim 100{ }^{\circ} \mathrm{C}$ está relacionada com a perda de umidade das amostras, na faixa $200-315{ }^{\circ} \mathrm{C}$ tem-se a degradação da hemicelulose, já a degradação térmica da celulose acontece em uma faixa mais alta de temperatura $\left(315-400{ }^{\circ} \mathrm{C}\right) \mathrm{com}$ pico de degradação $\sim 355^{\circ} \mathrm{C}$, enquanto a lignina é o componente que apresenta maior dificuldade de degradação, a perda de massa ocorre em um amplo intervalo de temperatura $\left(160-900^{\circ} \mathrm{C}\right)$. 
Tabela 1: Perda de massa (em \%) em função da temperatura e $\mathrm{T}_{\text {pico }}\left({ }^{\circ} \mathrm{C}\right)$.

\begin{tabular}{ccccccc}
\hline & \multicolumn{7}{c}{ Perda de Massa (\%) / temperatura $\left({ }^{\circ} \mathbf{C}\right)$} & $\mathbf{T}_{\text {pico }}$ \\
\hline Fibra & $\mathbf{1 0 0}{ }^{\circ} \mathbf{C}$ & $\mathbf{2 0 0}{ }^{\circ} \mathbf{C}$ & $\mathbf{3 0 0}^{\circ} \mathbf{C}$ & $\mathbf{~}^{\circ} \mathbf{C}$ & $\mathbf{5 0 0}^{\circ} \mathbf{C}$ & $\left({ }^{\circ} \mathbf{C}\right)$ \\
\hline $\mathrm{F}_{\mathrm{NT}}$ & 3,5 & 3,5 & 9,8 & 82,5 & 85,8 & 355 \\
$\mathrm{~F}_{\mathrm{T} 1}$ & 4,7 & 4,8 & 8,1 & 68,0 & 71,3 & 352 \\
$\mathrm{~F}_{\mathrm{T} 2}$ & 5,1 & 5,3 & 8,0 & 54,4 & 57,9 & 353 \\
\hline
\end{tabular}

A Figura 4a e b mostra as curvas TGA/DTG para os compósitos reforçados com 10 e $20 \%$ (m/m) de fibras em relação ao ABS puro. A estabilidade térmica do ABS puro foi de $365^{\circ} \mathrm{C}$. Para os compósitos de fibras $10 \% / \mathrm{ABS}$, a estabilidade térmica foi em torno de $310{ }^{\circ} \mathrm{C}$ e para os compósitos fibras $20 \% / \mathrm{ABS}$ foi cerca de $305^{\circ} \mathrm{C}$. A estabilidade térmica do ABS foi superior aos compósitos. Foi observado que com o aumento da adição de fibras a estabilidade térmica diminui. Este fato pode ser explicado devido ao excesso de fibras, elas não conseguem dispersar na matriz ocorrendo assim aglomeração e consequentemente, diminuição da estabilidade.
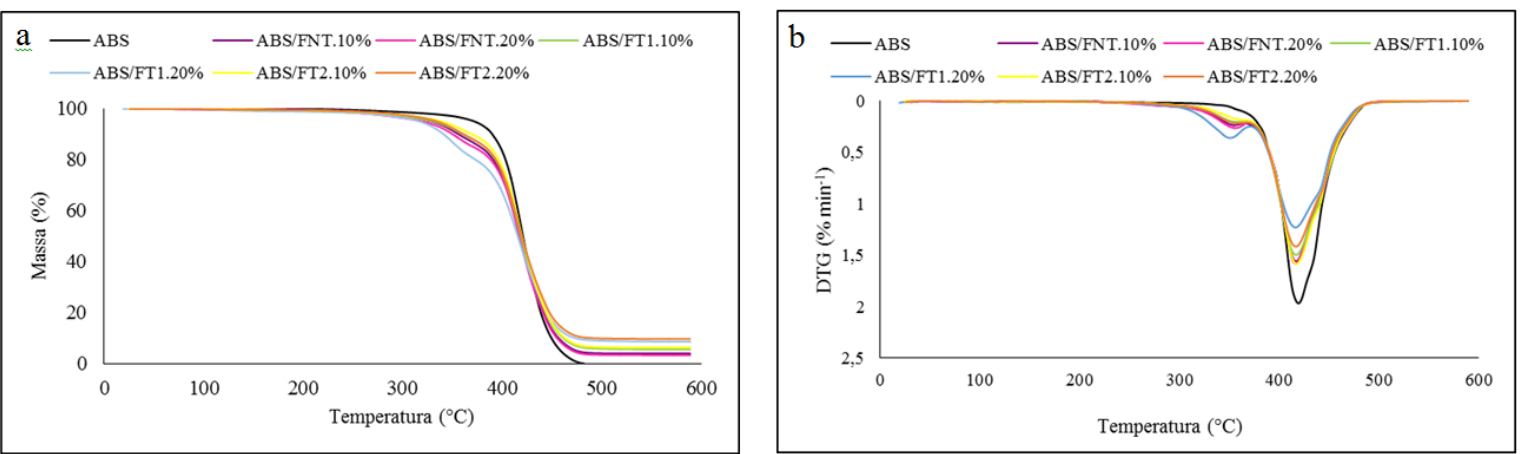

Figura 4: a) curvas termograviméticas (TGA); b) curvas de derivada termogravimétrica (DTG) para o ABS e compósitos.

As curvas DTG dos compósitos apresentaram dois picos distintos,já o ABS puro apresenta somente um pico, que podem ser observados na Figura 4b. A curva DTG do ABS apresentou $\mathrm{T}_{\text {pico }}$ em $420{ }^{\circ} \mathrm{C}$, com $\mathrm{T}_{\text {onset }}$ em $365{ }^{\circ} \mathrm{C}$; e para os compósitos defibras $10 \% / \mathrm{ABS}$ foi observado: o $\mathrm{ABS} / \mathrm{F}_{\mathrm{NT} .10 \%}$ apresentouT $\mathrm{T}_{\text {pico }}$ em $415^{\circ} \mathrm{C}$ e $\mathrm{T}_{\text {onset }}$ em $308{ }^{\circ} \mathrm{C}$, o $\mathrm{ABS} /$ $\mathrm{F}_{\mathrm{T} 1.10 \%}$ apresentouT $\mathrm{T}_{\text {pico }}$ em $416^{\circ} \mathrm{C}$ e $\mathrm{T}_{\text {onset }}$ em $311^{\circ} \mathrm{C}$ e o $\mathrm{ABS} / \mathrm{F}_{\mathrm{T} 2.10 \%}$ apresentouT $\mathrm{T}_{\text {pico }}$ em $417{ }^{\circ} \mathrm{C}$ e $\mathrm{T}_{\text {onset }}$ em $312{ }^{\circ} \mathrm{C}$; para compósitos defibras $20 \% / \mathrm{ABS}$ : o $\mathrm{ABS} / \mathrm{F}_{\mathrm{NT} .20 \%}$ apresentou $\mathrm{T}_{\text {pico }}$ em $415^{\circ} \mathrm{C}$ e $\mathrm{T}_{\text {onset }}$ em $305{ }^{\circ} \mathrm{C}$, o $\mathrm{ABS} / \mathrm{F}_{\mathrm{T} 1.20 \%}$ apresentouT em $415^{\circ} \mathrm{Ce} \mathrm{T}_{\text {onset }}$ em $305^{\circ} \mathrm{C}$ e o ABS $/ \mathrm{F}_{\mathrm{T} 2.20^{\circ}}$ apresentouT $\mathrm{T}_{\text {pico }}$ em $417^{\circ} \mathrm{Ce} \mathrm{T}_{\text {onset }}$ em $310^{\circ} \mathrm{C}$. Logo, entre os compósitos o que apresentou a temperatura de início de degradação mais elevada foi o $\mathrm{ABS} / \mathrm{F}_{\mathrm{T} 2.10 \%}\left(312^{\circ} \mathrm{C}\right)$, apresentando melhor estabilidade térmica entre as combinações testadas.

Com base na Tabela 2 observa-se que a amostra do polímero ABS sofreu total degradação a $500{ }^{\circ} \mathrm{C}$, os compósitos que apresentaram maior quantidade de resíduos à $600^{\circ} \mathrm{C}$ foram o $\mathrm{ABS} / \mathrm{F}_{\mathrm{T} 1.20 \%}(\sim 9 \%)$ e o $\mathrm{ABS} / \mathrm{F}_{\mathrm{T} 2.20 \%}(\sim 10 \%)$, logo percebese que os compósitos com maior quantidade de resíduo foram aqueles confeccionados com as fibras tratadas e com a proporção de $20 \%$ de fibra na matriz, por ter maior quantidade de fibras modificadas em consequência há maior quantidade de nanopartículas de sílica nesses compósitos, acarretando em aumento da massa residual.

Tabela 2: Perda de massa percentual em função da temperatura para o ABS e para os compósitos.

\begin{tabular}{|c|c|c|c|c|c|c|}
\hline \multirow[b]{2}{*}{ Tratamento } & \multicolumn{5}{|c|}{ Perda de Massa $(\%) /$ temperatura } & \multirow{2}{*}{$\begin{array}{l}\mathrm{T}_{\text {pico }} \\
\left({ }^{\circ} \mathrm{C}\right)\end{array}$} \\
\hline & $100^{\circ} \mathrm{C}$ & $200^{\circ} \mathrm{C}$ & $300^{\circ} \mathrm{C}$ & $400^{\circ} \mathrm{C}$ & $500^{\circ} \mathrm{C}$ & \\
\hline ABS & 0,04 & 0,09 & 1,38 & 17,00 & 100,00 & 420 \\
\hline $\mathrm{ABS} / \mathrm{F}_{\mathrm{NT} .10 \%}$ & 0,07 & 0,34 & 2,80 & 26,67 & 95,93 & 415 \\
\hline $\mathrm{ABS} / \mathrm{F}_{\mathrm{NT} .20 \%}$ & 0,40 & 0,86 & 3,65 & 27,85 & 96,53 & 415 \\
\hline $\mathrm{ABS} / \mathrm{F}_{\mathrm{T} 1.10 \%}$ & 0,13 & 0,69 & 2,87 & 25,58 & 94,15 & 416 \\
\hline $\mathrm{ABS} / \mathrm{F}_{\mathrm{T} 1.20 \%}$ & 0,38 & 1,14 & 3,55 & 33,08 & 90,99 & 415 \\
\hline $\mathrm{ABS} / \mathrm{F}_{\mathrm{T} 2.10 \%}$ & 0,06 & 0,61 & 2,49 & 22,85 & 93,54 & 417 \\
\hline $\mathrm{ABS} / \mathrm{F}_{\mathrm{T} 2.20 \%}$ & 0,24 & 0,60 & 2,51 & 25,29 & 90,09 & 417 \\
\hline
\end{tabular}




\subsection{Densidade do ABS e dos compósitos}

A Figura 5 apresenta os valores médios das densidades dos compósitos preparados. Observa-se que a adição de fibras na matriz de ABS provocou alteração na densidade do material. Adicionalmente, em todos os compósitos a inclusão de fibras acarretou em leve aumento da densidade quando comparadas com o ABS puro. Para o compósito $\mathrm{ABS} / \mathrm{F}_{\mathrm{T} 2.20 \%}$ foi observado o maior valor médio de densidade, o aumento da densidade desse material está relacionado com o maior incremento de massa ocasionada pela agregação de nanopartículas de sílica na superfície das fibras polpa celulose durante o processo de modificação para este tratamento $\left(\mathrm{F}_{\mathrm{T} 2}\right)$.

Neher et al. [15], em seu estudo com compósitos de ABS reforçado com fibras de palmeira, observaram que a adição de 5\% de fibras ocasionou a diminuição da densidade em relação ao ABS puro e a adição de teores maiores, $10 \%$ e $20 \%$, contribuíram para o aumento da densidade.

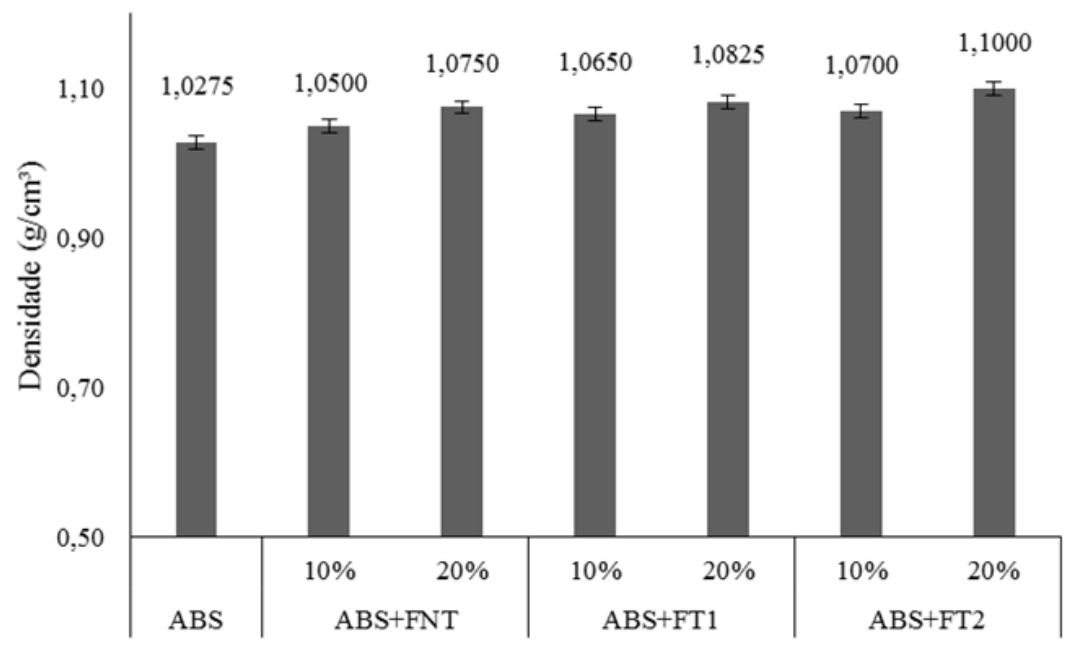

Figura 5: Valores médios de densidade $\left(\mathrm{g} / \mathrm{cm}^{3}\right)$ para o $\mathrm{ABS}$ e para os compósitos.

\subsection{Temperatura de deflexão térmica (HDT) e temperatura de amolecimento VICAT do ABS e compósitos}

A adição de fibras no polímero ABS acarretou em aumento da temperatura de deflexão térmica (HDT) para todos os compósitos testados em comparação com a HDT do ABS puro (Figura 6), sendo que o compósito que apresentou o maior valor de temperatura de deflexão térmica foi o $\mathrm{ABS} / \mathrm{F}_{\mathrm{T} 2.20^{\circ}}\left(78,13^{\circ} \mathrm{C}\right)$, aproximadamente $10^{\circ} \mathrm{C}$ superior ao $\mathrm{ABS}\left(68,89^{\circ} \mathrm{C}\right)$. Com relação ao teor de fibras na matriz observa-se que para cada tratamento os compósitos constituídos por $20 \%$ de fibras apresentaram HDT mais elevada do que para os compósitos com $10 \%$ de fibras.

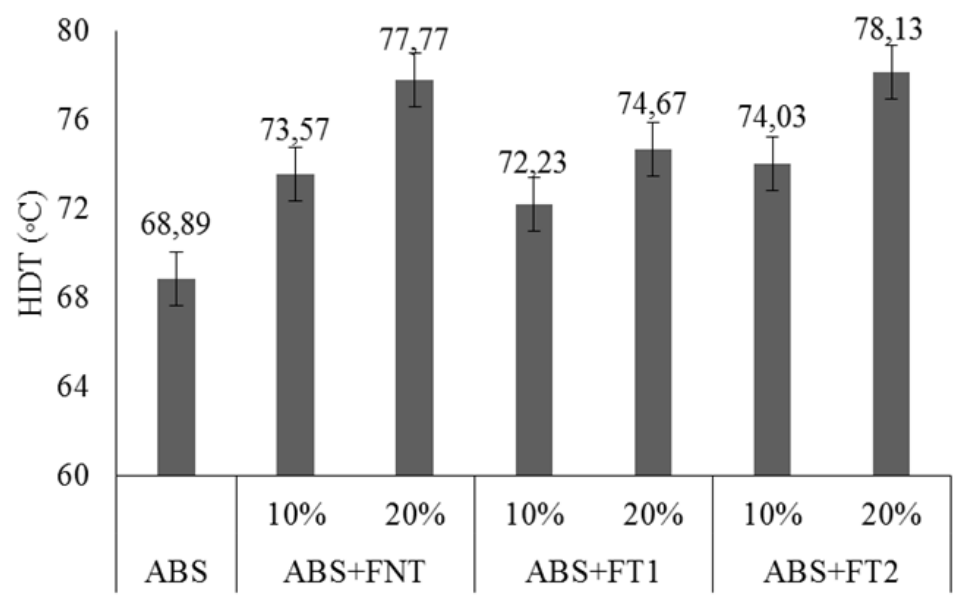

Figura 6: Valores médios de temperatura de deflexão térmica HDT $\left({ }^{\circ} \mathrm{C}\right)$ para o $\mathrm{ABS}$ e para os compósitos.

A temperatura de deflexão térmica (HDT) corresponde a temperatura em que uma quantidade arbitrária de deflexão ocorre, sob efeito de uma carga pré-determinada e a temperatura de amolecimento VICAT corresponde à temperatura na 
qual uma agulha penetra $1 \mathrm{~mm}$ no corpo de prova, sob efeito de carga específica [16]. Os ensaios de ponto de amolecimento e de deflexão térmica servem para fornecer uma margem de temperatura de segurança no uso de objetos plásticos, no entanto esses dados devem ser interpretados com ressalvas, considerando que na prática o material é submetido a cargas contínuas que levam os polímeros a sofrerem uma acelerada deformação [17].

Todos os compósitos apresentaram aumento de temperatura de amolecimento VICAT quando comparados com o ABS (Figura 7) enquanto, que o compósito que apresentou o maior valor foi o $\mathrm{ABS} / \mathrm{F}_{\mathrm{NT} .20 \%}\left(96,20{ }^{\circ} \mathrm{C}\right)$ seguido pelo compósito $\mathrm{ABS} / \mathrm{F}_{\mathrm{T} 2.20 \%}\left(95,43^{\circ} \mathrm{C}\right)$. Assim como para HDT observa-se que teor de fibras na matriz influenciou a temperatura de amolecimento VICAT, para cada tratamento os compósitos constituídos por $20 \%$ de fibras apresentaram VICAT mais elevadado que para os compósitos com $10 \%$ de fibras.

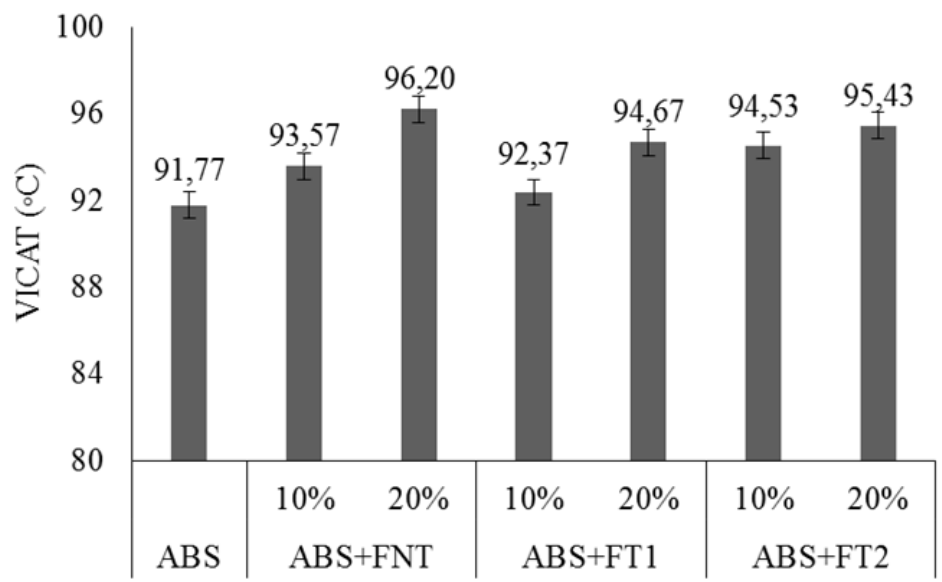

Figura 7: Valores médios de temperatura de temperatura de amolecimento VICAT $\left({ }^{\circ} \mathrm{C}\right)$ para o ABS e para os compósitos.

\section{CONCLUSÕES}

Pode-se concluir que a modificação das fibras foi bem-sucedida, comprovada pelas micrografias, sendo possível visualizar a agregação e distribuição das nanopartículas de sílica na superfície das fibras de polpa celulose. A modificação das fibras proporcionou incremento de massa e também favoreceu a estabilidade térmica das fibras de polpa celulose, aumentando a temperatura de início de degradação quando comparada com as fibras não modificadas, corroborando com os resultados obtidos por estudos semelhantes, possibilitando a utilização dessas fibras em condições que exijam maior estabilidade térmica.

Com relação a estabilidade térmica do compósito, tanto as fibras de polpa celulose não modificadas $\left(\mathrm{F}_{\mathrm{NT}}\right)$ quanto as modificadas $\left(\mathrm{F}_{\mathrm{T} 1}\right.$ e $\left.\mathrm{F}_{\mathrm{T} 2}\right)$, provocaram a diminuição da estabilidade dos compósitos com relação ao ABS, e os compósitos confeccionados com as fibras modificadas e com maior teor de fibra na matriz apresentaram maior quantidade de resíduo. Com relação ao percentual de fibra na matriz, têm-se que os compósitos com $20 \%$ de fibras apresentaram estabilidade térmica inferiores aos compósitos com $10 \%$. A adição de fibras de polpa celulose no terpolímero ABS acarretou em leve aumento da densidade dos compósitos. De modo geral a adição de fibras contribuiu para o aumento da temperatura de deflexão térmica (HDT) e da temperatura de amolecimento VICAT dos compósitos com relação ao ABS, aumentando a temperatura de aplicação desse material. O ganho nessas propriedades foi mais acentuado nos compósitos com teor de 20\% de fibras.

\section{AGRADECIMENTOS}

Os autores agradecem ao Laboratório de Microscopia e Microanálise (LMM) do Instituto de Ciências Biológicas (IB - UnB) pelas imagens de MEV, ao Laboratório de Polímeros da Universidade de Caxias do Sul (LPOL-UCS) onde os compósitos foram manufaturados, à Fundação de Apoio à Pesquisa do Distrito Federal (FAPDF) pelo apoio financeiro para a execução deste trabalho e à Coordenação de Aperfeiçoamento de Pessoal de Nível Superior (Capes) pela concessão da bolsa de mestrado ao primeiro autor

\section{BIBLIOGRAFIA}

[1] HARRIS, B., Engineering Composite Materials, The Institute of Materials, London, 1999. 
[2] TAJ, S., MUNAWAR, M. A., KHAN, S., Natural fiber-reinforced polymer composites, Proc. Pakistan Acad. Sci., v. 44, n. 2, pp. $129-144,2007$.

[3] MÜLLER, R. L., MÄHLMANN, C. M., RODRIGUEZ, A. L.; et al. Compósito de polietileno de baixa densidade e fibra de bagaço de cana-de-açúcar, Anais do $9^{\circ}$ Congresso Brasileiro de Polímeros, 2007.

[4] BARTON, J., NIEMCZYK, A., CZAJA, K., et al. Polymer composites, biocomposites and nanocomposites. Production, composition, properties and application fields, CHEMIK, v. 68, n. 4, pp. 280-287, 2014.

[5] CHEUNG, H.; HO, M., LAU, K., et al. Natural fibre-reinforced composites for bioengineering and environmental engineering applications, Composites: Part B, n. 40, pp.655-663, 2009.

[6] KUMAR, K. K., BABU, P. R., REDDY, K. R. N., Evaluation of Flexural and Tensile Properties of Short KenafFiber Reinforced Green Composites, International Journal of Advanced Mechanical Engineering, V. 4, N. 4, pp. 371-380, 2014

[7] VALADEZ-GONZALEZ, A., CERVANTES-UC, J. M., OLAYO, R., et al. Effect of fiber surface treatment on the fibermatrix bond strength of natural fiber reinforced composites, Composites: Part B, n. 30, pp.309-320, 1999.

[8] KADDAMI, H., DUFRESNE, A., KHELIFI, B., et al. Short palm tree fibers - Thermoset matrices composites, Composites: Part A, n. 37, pp. 1413-1422, 2006.

[9] POCAI, K., HILIG, E., FREIRE, E., ZENI, M., Estudo da influência de agente de acoplamento em compósitos de hdpe/ serragem, Anais do $8^{\circ}$ Congresso Brasileiro de Polímeros, 2005.

[10] RAABE, J., FONSECA, A. S., BUFALINO, L., et al. Evaluation of reaction factors for deposition of silica (SiO2) nanoparticles on cellulose fibers, Carbohydrate Polymers, v. 114, pp. 424-431, 2014.

[11] EVTUGUIN, D. V., PORTUGAL, I., GAMELAS, J. A. F., Modificação de pastas celulósicas com revestimentos à base de sílica, XXII TECNICELPA - Conferência Internacional da Floresta, Pasta e Papel, Tomar, Portugal, 2-4 de Outubro de 2013 .

[12] DIFALLAH, B. B., KHARRAT, M., DAMMAK, M., et al. Mechanical and tribological response of ABS polymer matrix filled with graphite powder, Materials and Design, v. 34, pp. 782-787, 2012.

[13] PINTO, R. J. B., MARQUES, P. A. A. P., BARROS-TIMMONS, A. M., et al. Novel SiO2/cellulose nanocomposites obtained by in situ synthesis and via polyelectrolytes assembly, Composites Science and Technology, 68, 1088-1093, 2008.

[14] YANG, H., YAN, R., CHEN, H., et al. Characteristics of hemicellulose, cellulose and lignin pyrolysis, Fuel 86, pp. 1781-1788, 2007.

[15] NEHER, B., BHUIYAN, Md. M. R., KABIR, H., et al. Study of Mechanical and Physical Properties of Palm Fiber Reinforced Acrylonitrile Butadiene Styrene Composite, Materials Sciences and Applications, n. 5, pp. 39-45, 2014.

[16] WASCHBURGER, M. R., Compósito de Polipropileno com Nanocarga, Dissertação de mestrado, Programa de PósGraduação em Engenharia de Minas, Metalúrgica e de Materiais - PPGEM, Universidade Federal do Rio Grande do Sul, Porto Alegre, 2006.

[17] MILES, D. C., BRISTON, J. H., Tecnologia do Polímeros, Editora Polígono S. A., São Paulo, 1975.

\section{ORCID}

Emanuela Camargo de Barros Lustosa Cláudio Henrique Soares Del Menezzi Sandra Maria da Luz

Joabel Raabe

Luiz Carlos Correia de Jesus

Rosineide Miranda Leã

Ademir José Zattera https://orcid.org/0000-0002-0732-7503

https://orcid.org/0000-0003-3369-2392

https://orcid.org/0000-0002-2223-0021

https://orcid.org/0000-0002-6797-151X

https://orcid.org/0000-0003-1079-4003

https://orcid.org/0000-0002-4218-3445

https://orcid.org/0000-0002-2198-4323 